\title{
REVISTA
}

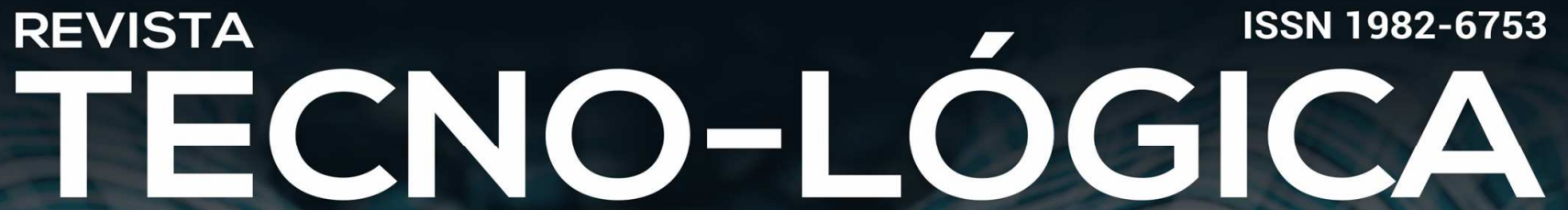

Edição Especial com publicação de trabalhos selecionados do V SIGEPRO

\section{MODELO CONCEITUAL DO ECOSSISTEMA DE VALOR DA MODA NA PERSPECTIVA DOS PRODUTOS WEARABLES}

Larissa Fortunati ${ }^{1}$, Rosiane Serrano ${ }^{1}$, Daniel Pacheco Lacerda ${ }^{2}$

${ }^{1}$ Instituto Federal de Educação, Ciência e Tecnologia do Rio Grande do Sul-Campus Erechim, 99713-028, Erechim, Brasil.

${ }^{2}$ Universidade do Vale do Rio do Sinos, 93022.750, Porto Alegre, Brasil.

*E-mail:larissafortunati@hotmail.com

Recebido em: 17/09/2020

Aceito em: 06/11/2020 DOI: 10.17058/tecnolog.v2i0.15954

\section{RESUMO}

O ecossistema de valor da moda é complexo e composto por múltiplos atores com interesses distintos e potencialmente conflitantes. Esta complexidade se amplifica ao introduzir novos processos ou produtos com o objetivo de promover a inovação tecnológica, tais como o desenvolvimento de artigos wearables (tecnologias vestíveis). Portanto, conhecer os atores pertencentes a este ecossistema é interessante. A presente pesquisa tem como objetivo apresentar os atores que compõem o ecossistema de valor da moda na perspectiva das tecnologias vestíveis (wearables technologies). Para tanto, realizou-se a revisão sistemática de literatura investigando estruturas de ecossistemas de valor, em segundo momento identificou-se os atores presentes nos estudos identificados e, por fim, propôsse o framework conceitual do ecossistema de valor. Como resultado, a pesquisa apresentou o modelo conceitual e inseriu a relação com indústrias como eletrônica e de software no ecossistema, além disso considerou os atores complementares, tais como os profissionais envolvidos no processo de produção do produto wearables.

Palavras-chave: Wearables Technologies. Tecnologias Vestíveis. Ecossistema de Valor. Moda.

\section{Introdução}

Os produtos wearables ou dispositivos vestíveis posicionam a tecnologia ao redor do corpo por meio de sensores [1] e possibilitam a interação usuário x ambiente, sem limitar os movimentos [2] durante atividades motoras e/ou cognitivas. Mann [3] apontou em seu estudo pregresso que no futuro os produtos wearables poderiam ser interconectados de modo a fornecerem informações para avalição médica, por exemplo.
A redução de custos e as funcionalidades dos dispositivos, fazem o mercado wearables crescer rapidamente [4], nos diversos setores industriais [5]. No setor de moda os dispositivos são encontrados em acessórios estéticos que podem ser utilizados no corpo ou sob ele, através de peças de roupa [67], não sendo uma categoria nova no mercado [5].

No entanto, informações sobre a presença destes produtos e tecnologias no processo produtivo do setor da moda ainda são limitadas e incertezas são frequentes [4] uma vez que 


\section{REVISTA}

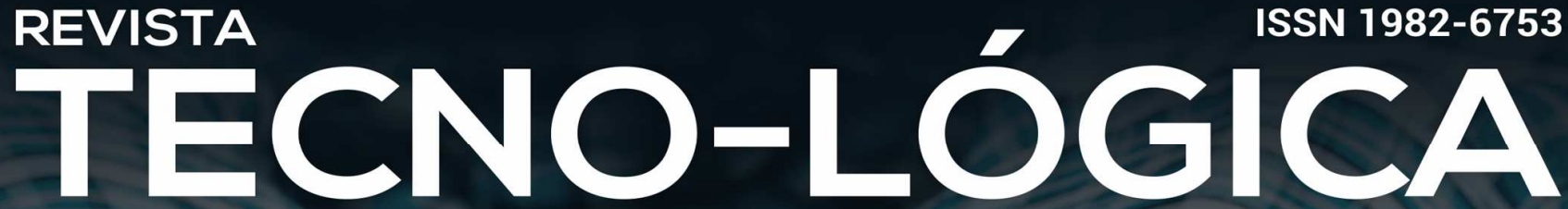

Edição Especial com publicação de trabalhos selecionados do V SIGEPRO

exige a interação com diversos setores industriais [8]. Por conseguinte, ao produzir um dispositivo vestível e adicionar funcionalidades neste é necessário inserir componentes técnicos [1] advindos de no mínimo dois setores industriais a moda e a eletrônica. Isso faz com que o fluxo de produção seja diferente dos processos convencionais [9].

Assim, a interação entre setores distintos produz um ecossistema composto por atores multidisciplinares que buscam desenvolver produtos e agregar valor para todos os participantes, gerando um processo de coevolução [10]. Portanto, identificar os atores e desenvolver estudos que considerem as relações entre estes, são importantes para ampliar a competitividade de um setor [11]. O setor da moda em especial, pois a sua competividade está atrelada ao desenvolvimento de produtos com diferentes níveis de tecnologia [11]. Bem como, é um setor intensivo em mão de obra e de baixa qualificação técnica e gerencial [12].

Destarte, o presente trabalho tem como objetivo apresentar os atores que compõem o ecossistema de valor da moda na perspectiva das tecnologias vestíveis (wearables technologies). Para tanto, incialmente foi revisada a literatura investigando estruturas de ecossistemas de valor, na sequência identificou-se os atores presentes em estudos prévios e, por fim, propôs-se o framework conceitual do ecossistema de valor da moda na perspectiva das tecnologias vestíveis. A revisão de literatura propicia uma visão abrangente e atual do tema em estudo, podendo confirmar, rejeitar, contrastar ou complementar resultados de pesquisas anteriores [13].

Esta pesquisa apresentou como resultado o modelo conceitual do ecossistema de valor da moda na perspectiva das tecnologias vestíveis, considerando a relação com indústrias como eletrônica e de software. Bem como, os atores complementares envolvidos no processo de produção do produto wearable, tais como como profissionais e os meios de distribuição.

O artigo é estruturado em 05 seções, sendo as primeiras a introdução e fundamentação teórica. Na sequência aborda-se a metodologia e os resultados da pesquisa. Por fim, as considerações finais do artigo.

\section{Ecossistemas de valor e a inserção das tecnologias vestíveis} no setor de moda

Empresas do setor industrial são relacionadas por Moore [10] como uma rede de organizações e indivíduos interconectados que formam um ecossistema [14] capaz de gerar um processo de coevolução. Portanto, definir um setor como um ecossistema possibilita ampliar a compreensão do ambiente de negócios [15] e verificar a agregação de valor resultante da interação destes para o desenvolvimento do setor.

Por conseguinte, o setor de moda pode ser identificado como um ecossistema, pois os atores com interesse distintos e potencialmente conflitantes, atuam de modo correlato visando o desenvolvimento do ecossistema como um todo. Identificam-se profissionais, produtores de matérias primas, compradores [16] e demais membros do ecossistema interessados em criar de valor para o negócio central [10].

Ao analisar o ecossistema de valor da moda na perspectiva dos produtos tecnológicos ou wearables, introduz-se atores advindos da computação e eletrônica, os quais deixam de operar como indústrias separadas e passam a atuar conjuntamente [8]. Com isso incertezas técnicas para a fabricação dos produtos wearables são amplificadas e mudanças no desenvolvimento da produção são frequentes [4]. 


\section{REVISTA}

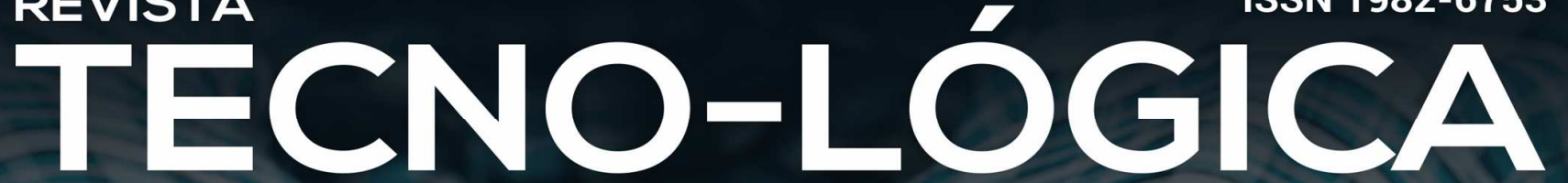

Edição Especial com publicação de trabalhos selecionados do V SIGEPRO

Além disso, a definição do tipo de wearable a ser projetado resulta em processos de produção distintos. Tais como, ao optar pela produção de um produto a partir da construção de circuitos eletrônicos ao redor do corpo ou embutidos em tecidos [1]. Na construção de circuitos os sensores eletrônicos ou dispositivos de computação são inseridos na peça de vestuário e está é considerada como suporte para o produto vestível. Ao integrar os dispositivos no tecido, o projeto inicia no desenvolvimento da fibra têxtil, na fabricação de tecido ou no estágio de acabamento [8]. Portanto, a produção de peças do vestuário com conceito vestível é um objeto de estudo [17].

Nessa perspectiva, inicialmente propõem-se explorar o ecossistema de valor da moda na perspectiva das tecnologias vestíveis (wearables technologies) identificando os atores necessários para o desenvolvimento deste produto. Uma vez que para produzir produtos competitivos, adequados às necessidades dos futuros clientes e aliados a melhores práticas de produção e redução dos impactos ambientais [5], deve-se conhecer os principais atores presentes no ecossistema em estudo. A próxima seção expõe a metodologia adotada para a condução desta pesquisa.

\section{Metodologia}

Ao definir a pesquisa como um meio para que a pessoas ampliem seu conhecimento sobre determinado assunto [18] torna-se necessário demonstrar os passos sistemáticos adotados para o seu desenvolvimento. Ao adotar estas práticas possibilita que a pesquisa seja contestada, verificada [19] e replicada. Para esta pesquisa foi utilizado como método de trabalho os passos descritos na Figura 1, os quais configuram o método de Revisão Sistemática da Literatura (RSL) [13].

TECNO-LÓGICA, Santa Cruz do Sul, v. 24, n. nesp, p. 368-378, jul./dez. 2020

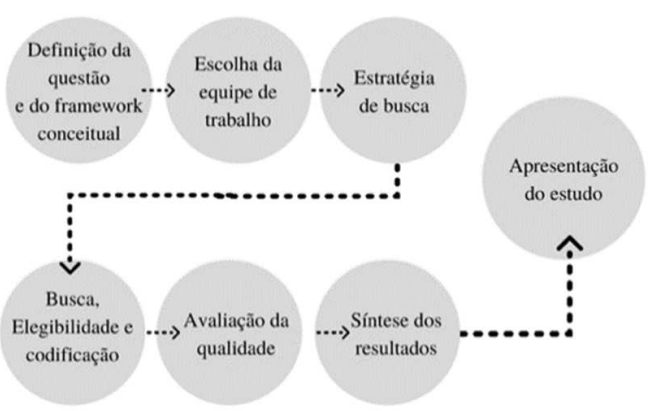

Figura 1 - Etapas para condução da Revisão Sistemática de Literatura. Fonte: Adaptado de Morandi e Camargo [13]

A definição da questão foi proposta a partir do interesse de identificar os atores pertencentes no ecossistema de valor da moda na perspectiva das tecnologias vestíveis (wearables technologies), pois em pesquisas prévias observou-se que a produção de peças do vestuário com conceito vestível é objeto de estudo [17]. O framework conceitual desta pesquisa é configurativo, pois as palavras-chave foram definidas a priori com relação ao tema de pesquisa.

A equipe de trabalho foi constituída por pesquisadores com expertise no tema e na abordagem metodológica adotada. A estratégia de busca iniciou pela definição das palavras-chave a serem pesquisadas, ou seja, "moda", "ecossistemas de valor", "cadeia de valor", "cadeia de suprimentos" e "tecnologias vestíveis", sendo estas em inglês e português. Segundo, as palavras-chave foram pesquisadas de modo combinado, utilizando o operador boleano "and". Terceiro, a pesquisa foi realizada nas bases de dados Ebsco, Scielo, Web of Science, Scopus, Emerald e na literatura cinzenta (Google Acadêmico).

Para a seleção dos documentos foram realizadas três análises, iniciando pelos títulos, na sequência verificou-se o conteúdo dos resumos e, por fim, os relevantes para leitura na íntegra foram armazenados. Como mostra a Tabela 1, foram

A matéria publicada nesse periódico é licenciada sob forma de uma Licença Creative Commons - Atribuição 4.0 Internacional http://creativecommons.org/licenses/by/4.0/ 


\section{REVISTA}

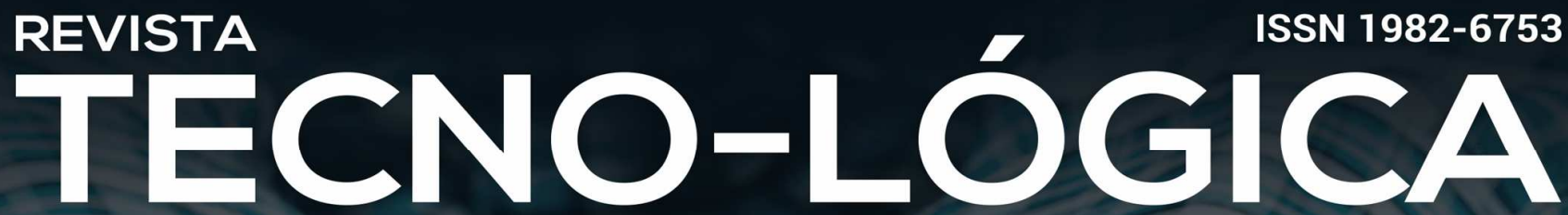

Edição Especial com publicação de trabalhos selecionados do V SIGEPRO

encontrados 6.881 títulos, sendo que 902 pesquisas (artigos científicos, relatórios técnicos, matérias de revistas) foram selecionadas para análise do resumo e 349 foram arquivadas para leitura na integra.

Tabela 1 - Resultados da Busca de documentos

\begin{tabular}{cccc}
\hline Base de dados & $\begin{array}{c}\text { Resultados } \\
\text { iniciais }\end{array}$ & $\begin{array}{c}\text { Abstracts } \\
\text { analisados }\end{array}$ & $\begin{array}{c}\text { Artigos } \\
\text { arquivados }\end{array}$ \\
\hline Scielo & 9 & 9 & 2 \\
Web of science & 24 & 24 & 19 \\
Scopus & 41 & 41 & 6 \\
EBSCO & 56 & 56 & 10 \\
Emerald & 53 & 53 & 17 \\
Google acadêmico & 6411 & 480 & 262 \\
Grey literature & 287 & 239 & 33 \\
TOTAL & $\mathbf{6 8 8 1}$ & $\mathbf{9 0 2}$ & $\mathbf{3 4 9}$ \\
\hline
\end{tabular}

A busca, elegibilidade e codificação considerou-se como critério de inclusão a aderência e a relevância com o tema. Os documentos que não expressavam sobre ecossistemas e tecnologias vestíveis foram considerados excluídos. A avaliação da qualidade dos estudos primários centrou-se no conteúdo do documento, ou seja, se estes apresentavam estruturas de ecossistemas de valor, cadeias de valor ou de suprimentos da moda. Foram identificados 13 estudos, os quais estão listados na Tabela 2 e embasaram a construção do ecossistema na perspectiva das tecnologias vestíveis (wearables technologies).

Para a construção da síntese dos resultados utilizou como técnica a crítica interpretativa e foi desenvolvido em três momentos, inicialmente pela separação dos materiais que apresentavam o tema de pesquisa. Em um segundo momento os documentos foram inseridos no software Atlas.ti e realizou-se uma codificação e categorização inicial dos elementos/atores presentes no ecossistema de valor da moda. Utilizou-se categorias e códigos definidos a priori e criados durante a leitura dos documentos. O uso do software para análise qualitativa permite a organização, gerenciamento de documentos [20], geração de resultados parciais das análises e possíveis reconfigurações de categorias e códigos.

A partir da análise inicial os elementos/atores identificados foram reorganizados e nomes similares foram agrupados nas categorias e códigos existentes. Os dados expostos em formato de matriz, como mostra a Tabela 3. A matriz expõe os atores identificados versus os estudos. A apresentação dos dados em matrizes facilita a visualização e permite a análise detalhada dos dados coletados [21] Por fim, a partir deste processo elaborou-se o ecossistema de valor da moda na perspectiva das tecnologias vestíveis. A próxima seção faz a apresentação do estudo.

\section{Resultados}

Esta seção expõem os resultados da Revisão Sistemática da Literatura e o modelo conceitual do ecossistema de valor da moda na perspectiva das tecnologias vestíveis (wearables technologies). Bem como, apresenta o modelo conceitual do ecossistema de valor.

\subsection{Análise preliminar dos estudos}

Após leitura preliminar dos 349 documentos, 13 estudos apresentaram estruturas de ecossistemas de valor, cadeias de valor ou de suprimentos da moda. Portanto, os documentos expostos na Tabela 2 foram inseridos no software Atlas.ti, onde a codificação e categorização dos elementos/atores presentes no ecossistema de valor da moda foi desenvolvida. 


\section{REVISTA}

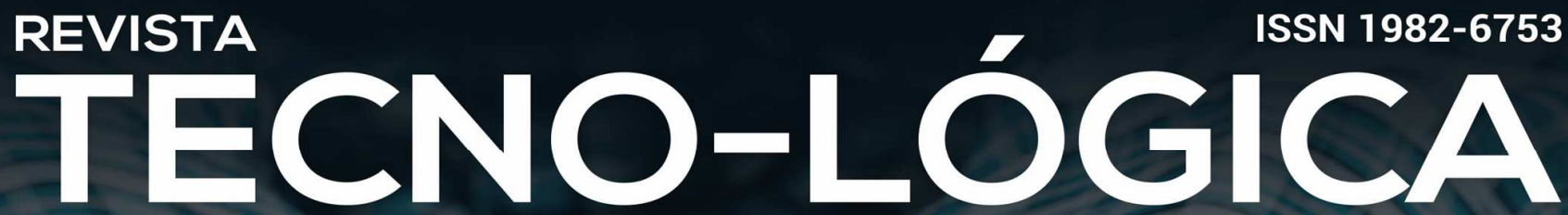

Edição Especial com publicação de trabalhos selecionados do V SIGEPRO

Tabela 2 - Relação de documentos analisados e utilizados

\begin{tabular}{|c|c|c|}
\hline $\mathbf{N}^{\circ}$ & Autores & Títulos dos Artigos \\
\hline 01 & EURATEX [22] & $\begin{array}{l}\text { European Technology Platform: for the future of } \\
\text { textiles and clothing- a vision for } 2020\end{array}$ \\
\hline 02 & Pinar & $\begin{array}{l}\text { Creating competitive advantage through } \\
\text { ingredient branding and brand ecosystem: The } \\
\text { case of Turkish cotton and textiles }\end{array}$ \\
\hline 03 & Strauss e & An NYCEDC Study \\
\hline 04 & Salon & $\begin{array}{l}\text { Bridging the Equity and Entrepreneurial Gaps in } \\
\text { the Finnish Fashion Industry }\end{array}$ \\
\hline 05 & Corner; Stric & $\begin{array}{l}\text { A local fashion ecosystem, the next step towards } \\
\text { an East London Fashion Cluster }\end{array}$ \\
\hline 06 & Mer & $\begin{array}{l}\text { Reconsidering the knowledge ecology in } \\
\text { fashion industry: A metaphorical approach }\end{array}$ \\
\hline 07 & $\begin{array}{l}\text { Fontell; Heikkilä } \\
\qquad[26]\end{array}$ & $\begin{array}{l}\text { Model of circular business ecosystem for } \\
\text { textiles }\end{array}$ \\
\hline 08 & $\mathrm{~W}$ & $\begin{array}{l}\text { Brief Analysis on Closed-loop Ecosystem of } \\
\text { Textile and Clothing Recycling }\end{array}$ \\
\hline 09 & Oksa & $\begin{array}{l}\text { In search of Finnish creative economy } \\
\text { ecosystems and their development needs-study } \\
\text { based on international benchmarking }\end{array}$ \\
\hline 10 & $\begin{array}{c}\text { Sandberg; Pal; } \\
\text { Hemilä [28] }\end{array}$ & $\begin{array}{l}\text { Exploring value creation and appropriation in } \\
\text { the reverse clothing supply chain }\end{array}$ \\
\hline 11 & Lin & $\begin{array}{l}\text { The structural characteristics of innovation } \\
\text { ecosystem: a fashion case }\end{array}$ \\
\hline 12 & Kaplanidou [30] & $\begin{array}{l}\text { Digitalization in the apparel manufacturing } \\
\text { process }\end{array}$ \\
\hline 13 & Chen [31] & $\begin{array}{l}\text { Value Creation by SMEs Participating in Global } \\
\text { Value Chains under Industry } 4.0 \text { Trend: Case } \\
\text { Study of Textile Industry in Taiwan }\end{array}$ \\
\hline
\end{tabular}

O estudo apresentado por EURATEX [22] foi utilizado como base para o desenvolvimento do ecossistema proposto por esta pesquisa. Este estudo foi definido como referência por apresentar os atores presentes no ecossistema de valor da moda e sua interligação, iniciando na indústria de extração e finalizando na reciclagem. O objetivo principal do estudo foi planejamento de estratégias futuras para cadeia de valor da moda, no que tange o acesso a recursos para o desenvolvimento de pesquisas e inovações EURATEX [22].

Nesta linha Oksanen et al. [14] analisam o ecossistema da economia criativa de produtos de vestuário em três países, com o intuito de fornecer recomendações para a indústria da moda. Fontell; Heikkilä [26] apresentam um ecossistema de negócios circular para têxteis e de vestuário, explicam os princípios da economia circular no contexto têxtil e os principais fluxos de materiais e tipos de atores existentes.

Strauss et al. [24] e Corner; Stride [25], por sua vez, evidenciam as indústrias locais como possíveis centros globais de moda, onde a promoção de empregos, treinamentos e espaços de trabalho podem auxiliar e informar os interessados no futuro da indústria da moda. O estudo de Pinar e Trapp [23] assinala que estratégias para promoção de marca e diferenciação de produto, podem promover o aumento da competitividade dos produtos têxteis.

A cadeia reversa de vestuário é apontada como um meio para demonstrar a sustentabilidade no ecossistema de valor da moda, como destacou Sandberg; Pal; Hemilä [28]. Assim, para Wang [27] a alteração na fabricação e no descarte de artigos de vestuário é uma solução para minimizar os impactos gerados na produção destes. Mengi [16] expõem que integração entre o segmento têxtil e de vestuário são oportunidades para criação de vantagens competitivas. Como apontado por Salonoja [15] a falta de integração e de colaboração entre as empresas de vestuário podem resultar no subdesenvolvimento do ecossistema e dificultar a obtenção de capital externo.

Para Chen [31] o uso da tecnologia é um fator relevante para empresas de pequeno porte na cadeia de suprimentos da moda. Lin [29], corrobora ao explorar a inovação tecnológica 


\section{REVISTA}

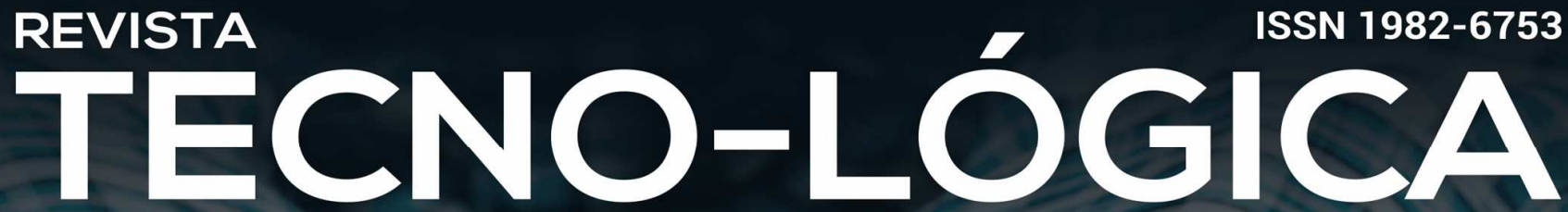

Edição Especial com publicação de trabalhos selecionados do V SIGEPRO

como meio para ampliar a imagem de um ecossistema de inovação. Por fim, Kaplanidou [30] demonstrou os aspectos e a influência da transformação digital em diferentes empresas do ramo de confecção, deste modo ressalta a necessidade de conhecimento sobre tecnologias digitais.

Como descrito na metodologia, os elementos/atores identificados foram analisados em dois momentos, o que possibilitou uma reorganização de nomes similares e agrupamento das categorias e códigos existentes. A Tabela 3 apresenta a matriz com analise desenvolvida, sendo atores versus estudos, os quais fundamentam o modelo conceitual do ecossistema de valor proposto.

\subsection{Modelo conceitual do ecossistema de valor da moda}

A partir destes elementos estruturou-se o modelo conceitual do Ecossistema de Valor na Moda na perspectiva das Tecnologias Vestíveis (Figura 2). O negócio central do ecossistema traz como elemento o "Produto de Vestuário Tecnológico". Este nome foi definido, pois entende-se ser necessário conhecer genericamente os atores presentes no ecossistema para assim, ao definir o produto a ser confeccionado, redirecionar os atores incluindo ou excluindo estes.

Observa-se que ao definir o negócio central torna-se necessário identificar as dimensões existentes no ecossistema, pois estas são responsáveis pelo seu funcionamento [10]. Neste estudo as dimensões são definidas como: Indústria de extração; Indústria de transformação; Indústria de bens/confecção, Varejo, Clientes, Cadeia reversa, Eletrônica. Além disso, observa-se a inserção de atores externos/apoio, os quais fornecem serviços, sendo definidos como: Distribuição, Profissionais e Outros.
A Indústria de extração é dividida em fibra natural e química, determina o tipo de tecido e os futuros processos de produção deste. A Indústria de transformação, converte as em fios e tecidos, por meio da tecelagem e do beneficiamento [22]. A Indústria de bens/confecção transforma o tecido em produtos de vestuário, podendo ser dividida em desenvolvimento, modelagem, corte e costura [16,30] e beneficiamento [22].

A dimensão Varejo representa os processos de venda físico ou virtual (on-line) [24,26,31]. A dimensão Clientes é expressa em convencionais, que buscam suprir suas necessidades individuais, e não convencionais, com o objetivo de suprir necessidades coletivas [22]. A Cadeia Reversa inserida no ecossistema representa o desuso e descarte dos produtos [22,24,26]. Por fim, a Eletrônica integra a informática e a nanotecnologia, responsável pela produção de componentes e peças que serão inseridos no produto tecnológico. A sua atuação dependerá da modalidade de produto a ser confeccionado, ou seja, se será integrada ao tecido ou no estágio de acabamento [8].

Os externos/apoio denominados Outros influenciam indireta ou diretamente o funcionamento do ecossistema como intermediários e negociadores, atuam na formulação de legislações, na infraestrutura e educação/cursos. Os Profissionais referem-se à atuação de agentes de apoio para o desenvolvimento dos produtos. A Distribuição refere-se à disponibilidade de serviços técnicos necessários para a produção do produto. Ambos, Profissionais e Distribuição, dividem-se em têxtil/confecção, serviços, provedores de tecnologia, provedores de conteúdo e transporte/logística. 


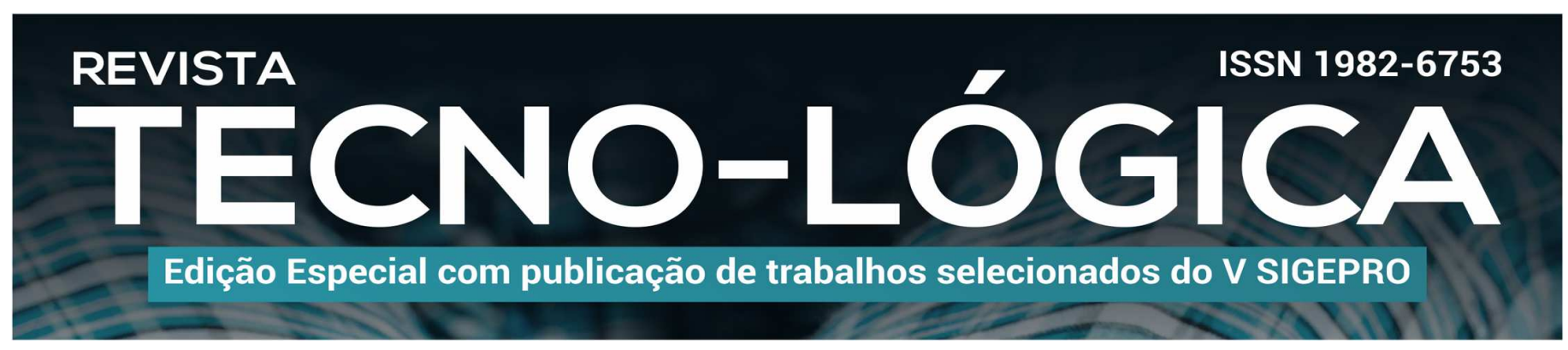

Assim, o modelo conceitual exposto na Figura 2 buscou representar o ecossistema de valor da moda na perspectiva das tecnologias vestíveis e a interligação dos atores presentes neste. A próxima seção aponta as considerações finais do estudo e as discussões.
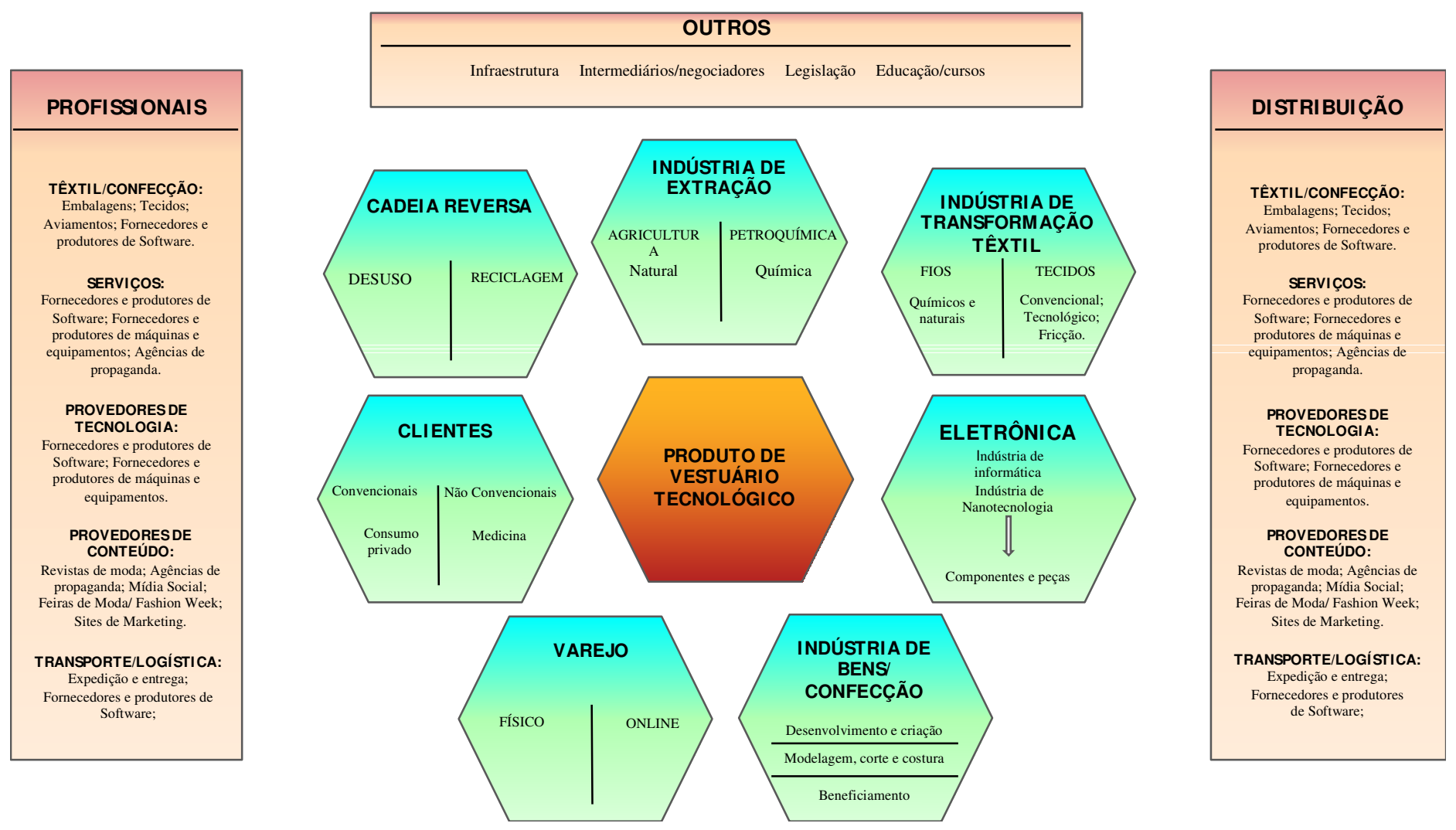

Figura 2 - Ecossistema de Valor da Moda na perspectiva das tecnologias vestíveis. 


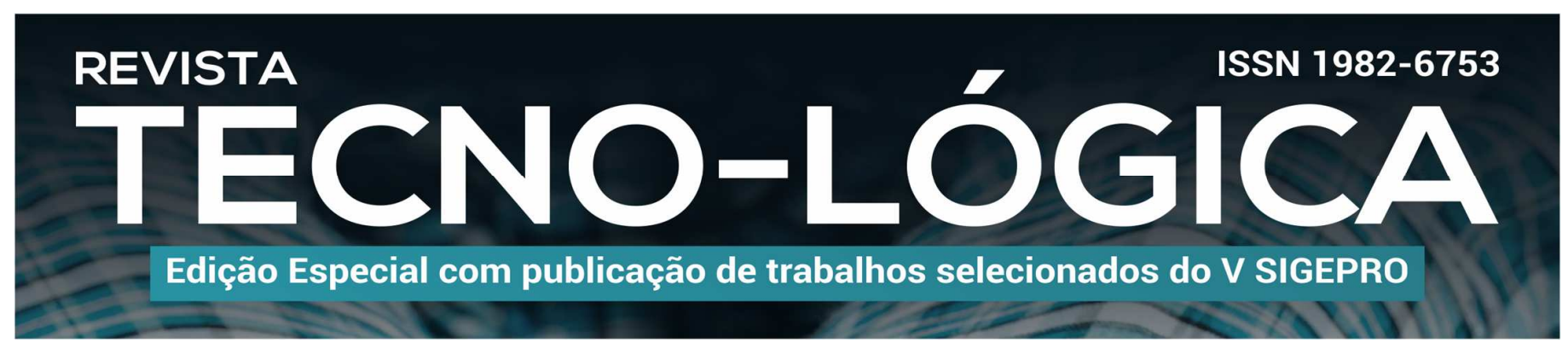

\begin{tabular}{|c|c|c|c|c|c|c|c|c|c|c|c|c|c|c|}
\hline \multicolumn{2}{|c|}{ Atores/Produtos } & \multirow{2}{*}{ 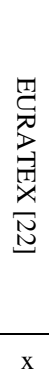 } & \multirow{2}{*}{ 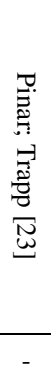 } & \multirow{2}{*}{ 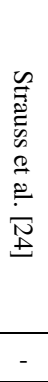 } & \multirow{2}{*}{ 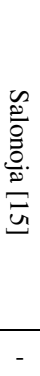 } & \multirow{2}{*}{ 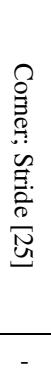 } & \multirow{2}{*}{ 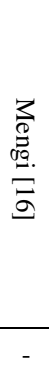 } & \multirow{2}{*}{ 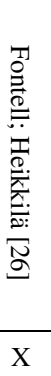 } & \multirow{2}{*}{ 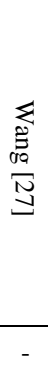 } & \multirow{2}{*}{ 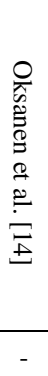 } & \multirow{2}{*}{ 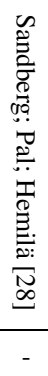 } & \multirow{2}{*}{$\begin{array}{l}\stackrel{5}{\Xi} \\
\stackrel{\mathbb{E}}{\Xi} \\
-\end{array}$} & \multirow{2}{*}{ 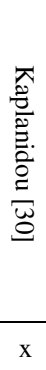 } & \multirow{2}{*}{$\begin{array}{c}\stackrel{\varrho}{\Xi} \\
\stackrel{\Xi}{\Xi} \\
-\end{array}$} \\
\hline Indústria de & Fibra Natural & & & & & & & & & & & & & \\
\hline extração & Fibra & $\mathrm{x}$ & - & - & - & - & - & - & - & - & - & - & - & - \\
\hline \multirow{9}{*}{$\begin{array}{l}\text { Indústria de } \\
\text { transformação } \\
\text { Têxtil }\end{array}$} & Indústria petroquímica & - & - & - & - & - & - & - & - & - & - & - & - & $\mathrm{x}$ \\
\hline & Indústria química & $\mathrm{x}$ & - & - & - & - & - & - & - & - & - & - & - & - \\
\hline & Indústria de fios químicos & $\mathrm{x}$ & - & - & - & - & - & $\mathrm{x}$ & $\mathrm{x}$ & - & - & - & $\mathrm{x}$ & $\mathrm{x}$ \\
\hline & Fábrica de tecelagem & - & - & - & - & - & - & - & $\mathrm{x}$ & - & - & - & $\mathrm{x}$ & $\mathrm{x}$ \\
\hline & Beneficiamento & $\mathrm{x}$ & - & - & - & - & - & - & - & - & - & - & - & - \\
\hline & Processamento industrial & - & - & - & - & - & - & $\mathrm{x}$ & - & - & - & - & - & - \\
\hline & Engomar e embalar & - & - & - & - & - & - & - & - & - & - & - & $\mathrm{x}$ & - \\
\hline & Expedição e entrega & - & - & - & - & - & - & - & - & - & - & - & $\mathrm{x}$ & - \\
\hline & Verificação e retrabalho & - & - & - & - & - & - & - & - & - & - & - & $\mathrm{x}$ & - \\
\hline \multirow{6}{*}{$\begin{array}{c}\text { Indústria de } \\
\text { bens/confecção }\end{array}$} & Costura & - & - & - & - & - & $\mathrm{x}$ & - & - & - & - & - & $\mathrm{x}$ & - \\
\hline & Fábrica de roupas & $\mathrm{x}$ & - & $\mathrm{x}$ & - & $\mathrm{x}$ & - & $\mathrm{x}$ & $\mathrm{x}$ & - & - & - & - & $\mathrm{x}$ \\
\hline & $\begin{array}{c}\text { Tingimento e acabamentos } \\
\text { (bordado, estampas) }\end{array}$ & - & - & $\mathrm{x}$ & - & - & - & - & - & - & - & - & - & - \\
\hline & Padronização e corte & - & - & - & - & - & - & - & - & - & - & - & $\mathrm{x}$ & - \\
\hline & Tecidos & $\mathrm{x}$ & - & - & - & - & - & $\mathrm{x}$ & - & - & - & - & - & - \\
\hline & Têxteis para o lar & $\mathrm{x}$ & - & - & - & - & - & - & - & - & - & - & - & - \\
\hline \multirow{4}{*}{ Distribuidores } & $\begin{array}{c}\text { Empresas de vestuário de } \\
\text { marca }\end{array}$ & - & - & - & - & - & - & - & - & - & $\mathrm{x}$ & $\mathrm{x}$ & - & - \\
\hline & Atacado & - & - & $\mathrm{x}$ & - & - & - & - & - & - & - & - & - & - \\
\hline & $\begin{array}{l}\text { Fornecedores de serviço/ } \\
\text { Materiais }\end{array}$ & $\mathrm{x}$ & $\mathrm{x}$ & - & - & - & - & - & - & $\mathrm{x}$ & $\mathrm{x}$ & $\mathrm{x}$ & - & $\mathrm{X}$ \\
\hline & $\ldots$ & $\ldots$ & $\ldots$ & $\ldots$ & $\ldots$ & $\ldots$ & $\ldots$ & $\ldots$ & $\ldots$ & $\ldots$ & $\ldots$ & $\ldots$ & $\ldots$ & $\ldots$ \\
\hline
\end{tabular}




\section{REVISTA}

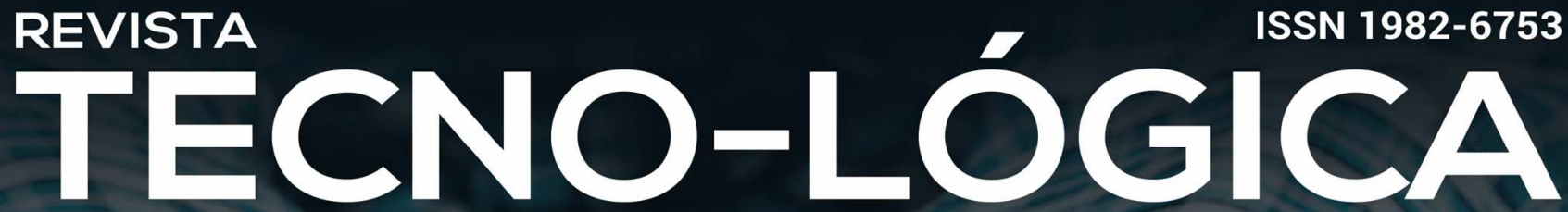

Edição Especial com publicação de trabalhos selecionados do V SIGEPRO

\section{Considerações finais e discussões}

Ao identificar os atores presentes no Ecossistema de Valor da Moda possibilitou evidenciar a complexidade deste setor, pois existe um elevado número de atores agindo de maneira integrada e simultânea, direta e indiretamente, visando a funcionalidade e alavancagem do setor. Isso pode ser observado nas etapas constantes na dimensão Indústria de bens/confecção. Observa-se que o objetivo proposto por esta pesquisa foi atingido, o qual foi identificar os participantes (atores) e elaborar um modelo de ecossistema de valor da moda na perspectiva das tecnologias vestíveis (wearables technologies).

A RSL, por sua vez, não apontou estudos que inserissem as tecnologias vestíveis em ecossistemas de valor da moda, cadeias de valor ou de suprimentos e sua relação com as demais indústrias. Isso é devido a necessidade de estudos aprofundados nesta temática [17]. A dimensão Eletrônica foi inserida considerando a necessidade de inserção de sensores ou atuadores nas peças de vestuários ou filamentos eletrônicos nos tecidos [1,8]. Além disso, a presença da Cadeia Reversa é exposta nos estudos, representando o reuso e descarte dos produtos [22,24,26]. Porém ao desenvolver um produto wearable o reuso e descarte deve ser explorado desde o início do processo de produção, pois os componentes eletrônicos e sensores inseridos não podem ser descartados inadequadamente.

Contudo, embora os resultados do estudo tenham se mostrado satisfatórios, limitações foram identificadas. A primeira refere-se à construção do modelo a partir dos artigos e documentos, ou seja, dados secundários. A segunda limitação refere-se a estrutura do ecossistema de valor apresentado, a qual foi seguida a temática de Moore [10] e foi dividida em negócio central, dimensões e atores externos. Portanto, o posicionamento dos atores pode não seguir a ordem adequada em decorrência da complexidade do setor. Propõem-se como trabalho futuro a validação do modelo conceitual apresentado nesta pesquisa, por meio de entrevistas com especialistas. Assim como a identificação e compreensão dos elementos alavancadores para o setor de moda na perspectiva das tecnologias vestíveis com o objetivo de gerar valor econômico, social e ambiental na medida em que este se desenvolve [11].

\section{Agradecimentos}

Os autores do artigo agradecem ao IFRS - Campus Erechim (Instituto Federal de Educação, Ciência e Tecnologia do Rio Grande do Sul - Campus Erechim) pelo suporte financeiro para o desenvolvimento deste trabalho.

\section{CONCEPTUAL MODEL OF FASHION VALUE ECOSYSTEM IN THE}

\section{PERSPECTIVE OF WEARABLES TECHNOLOGIES}

ABSTRACT: The clothing value ecosystem is complex and compose with multiples actors with distinct interests and potentially conflicting. This complexity is amplify when introduce new process or products with the goal to promote the technologic innovation, as the development of the articles wearables. Therefore, knowing the stakeholders to this ecosystem is interesting. The research has the goal to present the actors that compose the fashion value ecosystem in the perspective of wearables technologies. Thus, a systematic literature review was realized investigating structures of valuable ecosystems. In second moment was identified the actors in documents and finally, the conceptual framework of the value ecosystem was proposed. As the result of the research demonstrate the conceptual model of 


\section{REVISTA}

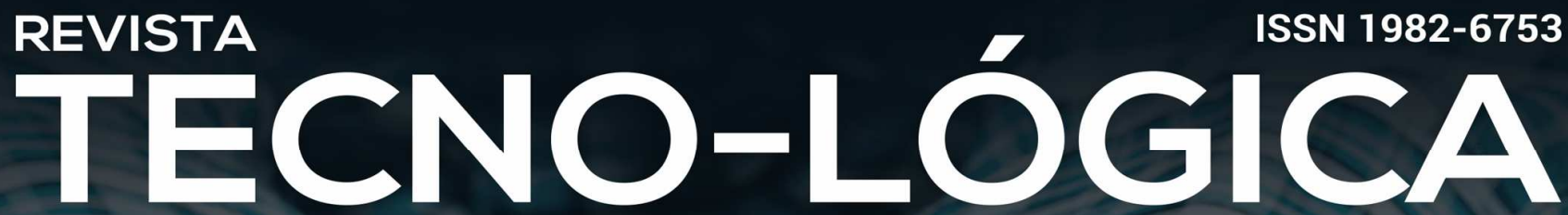

\section{Edição Especial com publicação de trabalhos selecionados do V SIGEPRO}

clothing ecosystem and your relations with the electronic and software in ecosystem. The study considered the complementary actors, as the professional's enrolment in production process of wearables.

Keywords: Wearables Technologies. Value ecosystem. Fashion.

\section{REFERÊNCIAS}

[1]. O’Nascimento R. Roupas Inteligentes: Combinando Moda e Tecnologia. São Paulo, Brasil: Editora Senac São Paulo, 2020.

[2]. Donati LP. Computadores Vestíveis: Convivência de Diferentes Especialidades The INmobility Project View Project. Caxias do Sul, 2004.

[3]. Mann S. Smart clothing: The wearable computer and wearcam. Pers Technol 1997;1:21-7.

[4]. Dehghani M, Kim KJ. Past and Present Research on Wearable Technologies: Bibliometric and Cluster Analyses of Published Research from 2000 to 2016. Int J Innov Technol Manag 2019;16:1950007.

[5]. Mardonova M, Choi Y. Review of Wearable Device Technology and Its Applications to the Mining Industry. Energies 2018;11:547.

[6]. Cantanhede LRC, Dias EJP, Gammarano I de JLP et al. COMPORTAMENTO DO CONSUMIDOR DE TECNOLOGIA VESTÍVEL: CARACTERÍSTICAS QUE INFLUENCIAM NA INTENÇÃO DE CONSUMO. REAd Rev Eletrônica Adm (Porto Alegre) 2018;24:244-68.

[7]. Lazaroiu G. Communicative functions of smart clothing. Contemp Readings L Soc Just Contemp Readings Law Soc Justice 2012;211:162-7.

[8]. Wood J. Revolutions in wearable technology for apparel. High-Performance Apparel. Manchester: Elsevier, 2018, 325-39.

[9]. Han SC, Han Y-H, Kim HS. Characteristics and perspectives of wearable smart devices and industrial ecosystem. 2014 20th IEEE International Conference on Parallel and Distributed Systems (ICPADS). Vol 2015-April. IEEE, 2014, 911-4.

[10]. Moore JF. The Death of Competition: Leadership and Strategy in the Age of Business Ecosystems. New York, Estados Unidos da América: HarperBusiness,

TECNO-LÓGICA, Santa Cruz do Sul, v. 24, n. nesp, p. 368-378, jul./dez. 2020
1996.

[11[. Serrano R, Rodrigues LH, Lacerda DP et al. Systems Thinking and Scenario Planning: Application in the Clothing Sector. Syst Pract Action Res 2018;31:509_ 37.

[12]. Antero SA. Articulação de políticas públicas a partir dos fóruns de competitividade setoriais: a experiência recente da cadeia produtiva têxtil e de confecções. Rev Adm Pública 2006;40:57-79.

[13]. Morandi MIWM, Camargo LFR. Revisão sistemática da literatura. Design Science Research: Método de Pesquisa Para Avanço Da Ciência e Tecnologia. Porto Alegre: Bookman, 2015, 141-75.

[14]. Oksanen J, Kuusisto O, Lima-Toivanen M et al. In search of Finnish creative economy ecosystems and their development needs-study based on international benchmarking. Publ Gov Anal Assess Res Act 50/2018 2018:155.

[15]. Salonoja N. Bridging the Equity and Entrepreneurial Gaps in the Finnish Fashion Industry. 2013:130.

[16]. Mengi O. Reconsidering the knowledge ecology in fashion industry: A metaphorical approach. Proc Eur Conf Knowl Manag ECKM 2017;2:683-9.

[17]. Eidenhammer L. Wearables conquering the Workplace of Generation Y-The opportunities and risks to integrate Wearable Technology at work. 2018.

[18]. Saunders M, Lewis P, Thornhill A. Research Methods for Business Students. 5th ed. Harlow, England: Pearson Education Limited, 2009.

[19]. Dresch A, Lacerda DP, Antunes Jr JAV. Design Science Research. A Method for Science and Technology Advancement. 1st ed. London: Springer, 2015.

[20]. Gibbs GR. Using Software in Qualitative Analysis. The SAGE Handbook of Qualitative Data Analysis. 1 Oliver's Yard, 55 City Road, London EC1Y 1SP United Kingdom: SAGE Publications Ltd, 2014, 277-94.

[21]. Miles MB, Huberman AM, Saldana J. Qualitative Data Analysis: A Methods Sourcebook. 3rd ed. Thousand Oaks, EUA: Sage Publications, Inc, 2013.

[22]. European Apparel and Textile Organisation - EURATEX. European Technology Platform: For the Future of Textiles and Clothing - a Vision for 2020. Brussels: European Apparel and Textile Organisation, 2004.

[23]. Pinar M, Trapp PS. Creating Competitive Advantage Through Ingredient Branding and Brand Ecosystem. J Int Food Agribus Mark 2008;20:29-56.

[24]. Strauss S, Sundjaja K, Johnson E et al. Fashion NYC 2020. New York: New 


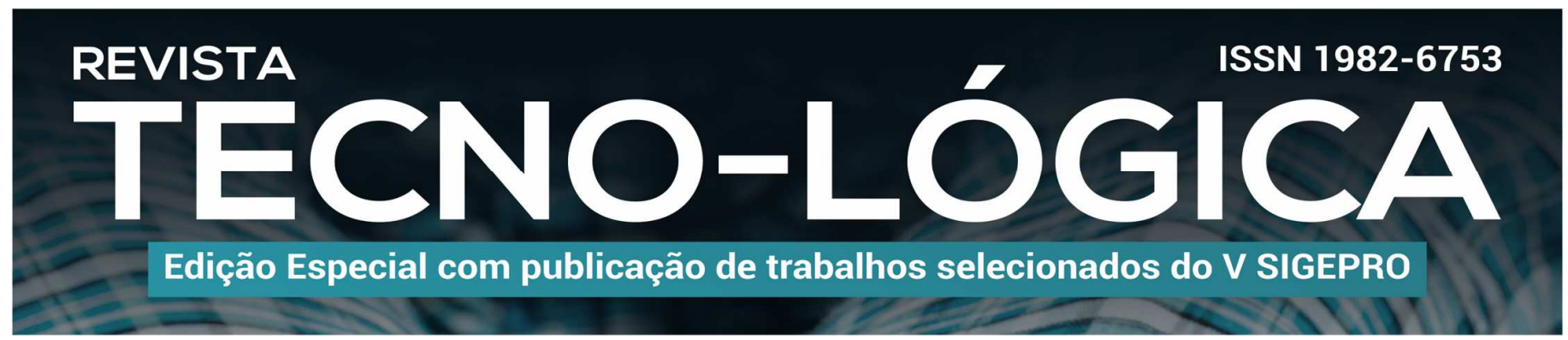

York City Economic Development Corporation, 2010

[25]. Corner F, Stride S. A Local Fashion Ecosystem, the next Step towards an East London Fashion Cluster. London: High-end \& Designer Manufacturing Report, Oxford Economics and Glasgow Caledonian University, 2015.

[26]. Fontell P, Heikkilä P. Model of circular business ecosystem for textiles. 2017:58.

[27]. Wang S. Brief Analysis on Closed-loop Ecosystem of Textile and Clothing Recycling. IOP Conf Ser Earth Environ Sci 2018;186:012058.
[28]. Sandberg E, Pal R, Hemilä J. Exploring value creation and appropriation in the reverse clothing supply chain. Int J Logist Manag 2018;29:90-109.

[29]. Lin S. The structural characteristics of innovation ecosystem: a fashion case. Eur J Innov Manag 2018;21:620-35.

[30]. Kaplanidou A. Digitalization in the apparel manufacturing process. 2018.

[31]. Chen C-L. Value Creation by SMEs Participating in Global Value Chains under Industry 4.0 Trend: Case Study of Textile Industry in Taiwan. J Glob Inf Technol Manag 2019;22:120-45. 